\title{
Endoscopic Transnasal Approach for Urgent Decompression of the Craniocervical Junction in Acute Skull Base Osteomyelitis
}

\author{
Terry C. Burns ${ }^{1}$ Stefan A. Mindea ${ }^{1} \quad$ Arjun V. Pendharkar ${ }^{1} \quad$ Nicolae B. Lapustea $^{1} \quad$ Ioana Irime ${ }^{1}$ \\ Jayakar V. Nayak ${ }^{2}$
}

${ }^{1}$ Department of Neurosurgery, Stanford University School of Medicine, Stanford, California, United States

2 Department of Otolaryngology-Head and Neck Surgery, Stanford University School of Medicine, Stanford, California, United States

\begin{abstract}
Address for correspondence Jayakar V. Nayak, MD, PhD, Department of Otolaryngology-Head and Neck Surgery, Stanford Sinus and Endoscopic Skull Base Center, 801 Welch Road, Stanford, CA 94305,
\end{abstract} United States (e-mail: jnayak@ohns.stanford.edu).

J Neurol Surg Rep 2015;76:e37-e42.

Abstract
Keywords
- craniocervical
junction osteomyelitis
- endoscopic transnasal
odontoidectomy
- extended endonasal
approach
- spine infection
- skull base
- C1
- odontoid
- dens

Ventral epidural abscess with osteomyelitis at the craniocervical junction is a rare occurrence that typically mandates spinal cord decompression via a transoral approach. However, given the potential for morbidity with transoral surgery, especially in the setting of immunosuppression, together with the advent of extended endonasal techniques, the transnasal approach could be attractive for selected patients. We present two cases of ventral epidural abscess and osteomyelitis at the craniocervical junction involving $\mathrm{C} 1 / \mathrm{C} 2$ that were successfully treated via the endoscopic transnasal approach. Both were treated in staged procedures involving posterior cervical fusion followed by endoscopic transnasal resection of the ventral $\mathrm{C} 1 \mathrm{arch}$ and odontoid process for decompression of the ventral spinal cord and medulla. Dural repairs were successfully performed using multilayered, onlay techniques where required. Both patients tolerated surgery exceedingly well, had brief postoperative hospital stays, and recovered uneventfully to their neurologic baselines. Postoperative magnetic resonance imaging confirmed complete decompression of the foramen magnum and upper C-spine. These cases illustrate the advantages and low morbidity of the endonasal endoscopic approach to the craniocervical junction in the setting of frank skull base infection and immunosuppression, representing to our knowledge a unique application of this technique to osteomyelitis and epidural abscess at the craniocervical junction.

\section{Introduction}

Pyogenic osteomyelitis of the craniocervical junction is a rare event that has been infrequently described in the literature. ${ }^{1-9}$ The clinical manifestations most often include neck pain, stiffness, swelling, and dysphagia, although this entity may also present with meningitis. Most commonly afflicted are elderly or immunocompromised patients harboring comorbidities such as diabetes or end-stage renal disease. In the setting of sepsis despite the use of broad-spectrum intravenous antibiotics, surgical debridement of infected vertebral bone may be required. In addition, ventral spinal cord impingement with atlantoaxial instability, fluid accumulation, and bony degeneration from septic arthritis may develop acutely, requiring urgent decompression. Surgical decompression of this site has traditionally been performed via the transoral approach. $2,10,11$ However, this technique is often associated with notable morbidities, including received

May 31, 2014 accepted after revision

August 14, 2014

published online

January 16, 2015
DOI http://dx.doi.org/

10.1055/s-0034-1395492. ISSN 2193-6366.
License terms

(c) 2015 Georg Thieme Verlag KG

Stuttgart · New York

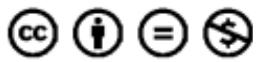


velopharyngeal insufficiency, postoperative dysphagia, and surgical bed contamination from saliva, oral flora, and food particles in the presence of active cervical spine infection. As such, the transoral approach can be particularly challenging for immunocompromised patients with poor baseline health status. We and others more recently described the transnasal endoscopic approach to the craniocervical junction including endoscopic transnasal odontoidectomy. ${ }^{12-24}$ To date, the application of this advanced procedure has remained limited to a few specialized academic surgical centers. However, the feasibility of this approach has been demonstrated in the treatment of atlantoaxial subluxation, rheumatoid pannus, and other mass lesions producing ventral medullary compression. Importantly, this approach is amenable to complete resection of the odontoid process, and has thus far been associated with highly favorable clinical outcomes. Management of cerebrospinal fluid (CSF) leak at the craniocervical junction, where primary dural repair is not possible, also represents a reconstructive challenge for this remote skull base defect, which may itself contribute to secondary brainstem compression. We present two cases utilizing the transnasal endoscopic approach for treatment of acute craniocervical junction osteomyelitis at the in the setting of frank immunosuppression. We further discuss methods for successful endoscopic repair of challenging CSF leaks in this rare location of the ventral skull base.

\section{Case 1}

\section{History}

A 69-year-old female patient with a medical history, including rheumatoid arthritis initially presented to her primary care provider with 3 weeks of progressive neck pain and upper/lower extremity paresis. Magnetic resonance imaging (MRI) revealed a dense $\mathrm{C} 1 / \mathrm{C} 2$ pannus with invagination of the thecal sac. After 1 day, she was admitted to a local hospital with altered mental status where further questioning revealed a history of low-grade fever and chills, odynophagia, diplopia, blurry vision, and photophobia. Upon transfer to our tertiary care institution, the patient's physical examination was notable for a right third nerve palsy, dense hemiparesis in the left upper and lower extremities, and fluctuating mental status with intermittent unresponsiveness. Laboratory values on admission demonstrated marked leukocytosis in both blood and CSF specimens and elevated inflammatory markers. Brain and cervical spine MRI revealed cerebritis, intraventricular regions of diffusion restriction indicative of purulence, and an enhancing collection at the atlantoaxial junction resulting in compression of the ventral spinal cord. Empiric intravenous antibiotics were initiated and blood cultures ultimately grew Streptococcus pneumoniae. The patient was initially deemed too unstable to undergo transoral decompression of the craniocervical junction, although her mental status gradually improved with antibiotics. Her left arm and leg weakness persisted and repeat MRI 5 days later redemonstrated an enhancing collection at the atlantoaxial junction concerning for epidural abscess with severe canal stenosis and intrinsic cervicomedullary cord-signal change (- Fig. 1A, B). A computed tomography (CT) scan demonstrated bony reaction and neo-osteogenesis at $\mathrm{C} 1 / 2$ (-Fig. 1C) suggestive of vertebral osteomyelitis. In light of an improvement in mental status, we elected to pursue transnasal surgical debridement and decompression of the craniocervical junction.

\section{Intervention}

Preoperative CT scan utilizing the hard palate suggested appropriately achieved surgical access via transnasal approach. CT angiography (-Fig. 1C) documented the course of the vertebrobasilar arteries and the hypoglossal canals for intraoperative navigation following posterior cervical fusion (see - Fig. 1D). After 2 days, an anterior transnasal debridement was performed as a combined procedure between neurosurgery and otolaryngology. After upfront placement of a lumbar drain, the transnasal approach included bilateral inferior turbinate outfractures and a posteroinferior septectomy. After adequate nasopharyngeal soft tissue removal, access to the posterior fossa skull base and atlantoaxial joint was obtained. Extended diamond bits were used to widely drill down the anterior arch of $\mathrm{C} 1$ from a lateral-to-medial direction, using intraoperative image guidance to confirm the course of the vertebral arteries. Abnormal fibrous and seemingly infected granulation tissue was noted within the $\mathrm{C} 1$ and C2 joint space that was subsequently resected. Following this, a diseased odontoid process was found to be associated with significant scarring and firmly adherent to the dura overlying the basilar cistern. Extensive drilling at the base of the odontoid process permitted anterior displacement and removal of the dens.

Although successful decompression was achieved, a $1.5 \mathrm{~cm}$ dural tear was clearly noted at the site of odontoid-dural tethering and displacement, leading to frank CSF drainage and ballooning of the dura ventrally into the surgical field. This provided a challenge given the remote surgical corridor of 13 to $14 \mathrm{~cm}$ posterior to the nostril rim, and the need to avoid brainstem compression in the process of bolstering a dural repair construct. We elected to proceed with a multilayered onlay technique, reserving the option of a nasoseptal flap for the setting of persistent fistula. DuraGen matrix (Integra LifeSciences Corp., Plainsboro Township, New Jersey, United States) was placed atop the dural defect, followed by abdominal fat graft placement, and Tisseel (Baxter Healthcare Corp., Illinois, Deerfield, United States). To close the sizeable dead space within the endoscopic corridor, a second layering consisting of fat, Gelfoam (Pfizer Corp., New York, New York, United States) and Tisseel was utilized. For final support of the reconstruction, resorbable Nasopore (Polyganics Corp., Groningen, The Netherlands) microfibrillar sponge was partially extended into the reconstruction, spanning across the soft palate, and onto the nasal floor. No evidence of CSF leak was detected upon multiple Valsalva maneuvers and lumbar CSF diversion was maintained at $15 \mathrm{~mL} / \mathrm{h}$.

The patient was extubated, and tolerated an oral diet on postoperative day 1. Postoperative imaging demonstrated complete anterior resection of $\mathrm{C} 1$ and $\mathrm{C} 2$ with brainstem decompression without evidence of secondary compression following reinforcement of the multilayered reconstruction 

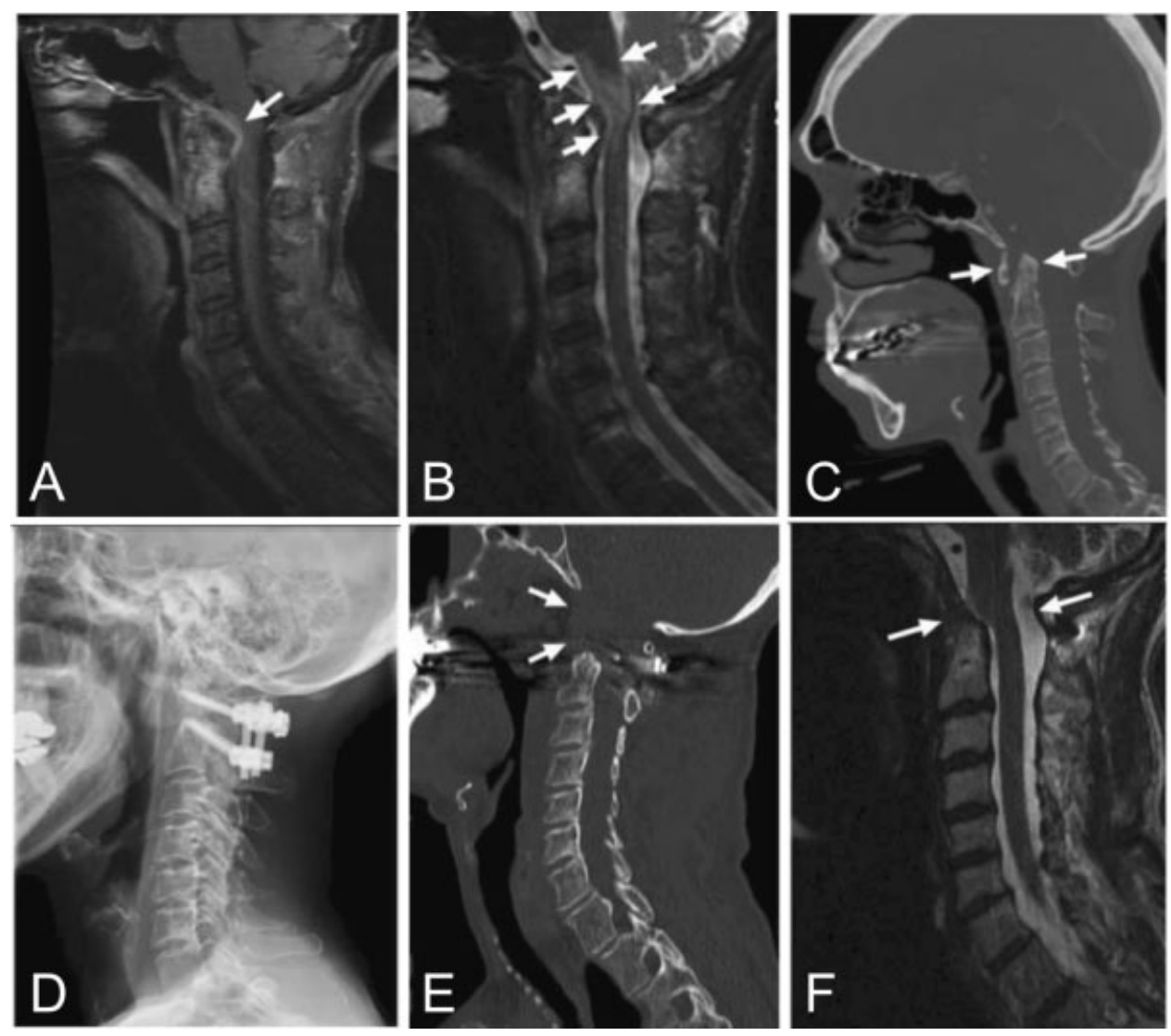

Fig. 1 Case 1: (A) Gadolinium enhancement posterior to the dens is seen with significant mass effect on the cevicomedullary junction. (B) Signal abnormality is seen in the upper cervical cord and medulla as well as bone on T2-weighted MRI. (C) Preoperative CT angiogram highlights bony changes, vascular structures (arrows), and unobstructed access to the atlantoaxial junction via the endonasal route. (D) C1-2 posterior instrumentation is seen on lateral X-ray. (E) Postoperative CT demonstrates the extent of bony resection achieved (arrows). (F) Postoperative T2weighted MRI confirms decompression of the cervicomedullary junction. CT, computed tomography; MRI, magnetic resonance imaging.

(-Fig. 1E, F). Coagulase-negative Staphylococcus grew from intraoperative cultures to direct future parenteral antibiotics. The patient was discharged on postoperative day 5 following discontinuation of CSF diversion.

\section{Follow-Up}

The patient made steady neurologic recovery without noting postoperative rhinorrhea, velopharyngeal insufficiency, or Eustachian tube dysfunction at any time. Endoscopic visualization of the surgical site 3 months postoperatively revealed a completely remucosalized nasopharynx without any gross evidence of surgical manipulation. Follow-up neurological examination 6 months after the surgery revealed $5 / 5$ strength in the previously weak upper extremity; no meningeal signs or rhinorrhea were present, and the posterior cervical incision was well healed. By this time the patient had returned to work as an administrative assistant.

\section{Case 2}

\section{History}

A 55-year-old male patient was transferred to our institution with progressive headaches, a "clicking, unstable" sensation in his neck, and a large, enhancing ventral epidural collection was noted to be impinging the cervicomedullary junction. His medical history included diabetes mellitus, hypertension, hyperlipidemia, and intravenous drug abuse. Few years ago, he had undergone a prior $\mathrm{C} 3-\mathrm{C} 7$ anterior fusion with C3-4 cage placement and C3-T1 posterior stabilization at an outside hospital for cervical spondylosis, he had a persistent C3-4 myelomalacia and walked with a cane at baseline. Upon transfer, the patient was afebrile and cognitively intact with no meningeal signs or focal weakness, though he had immunosuppression secondary to poorly controlled diabetes in the setting of intravenous (IV) drug use. Laboratory workup revealed elevated inflammatory markers, but no leukocytosis. Empiric broad-spectrum antibiotics were initiated. Preoperative MRI demonstrated a large, heterogeneously enhancing collection at the atlantoaxial articulation with effacement of the thecal sac ( - Fig. 2A, B). Additional imaging findings included bony thickening and neo-osteogenesis of the anterior arch of $\mathrm{C} 1$, the body of $\mathrm{C} 2$, and the base of the odontoid process (-Fig. 2C). Anterior and posterior instrumentation was present from C3-T1 with good bony fusion. In view of the patient's progressive symptoms, large enhancing cervicomedullary collection and relatively stable medical condition, he was deemed an appropriate candidate for surgical decompression. 

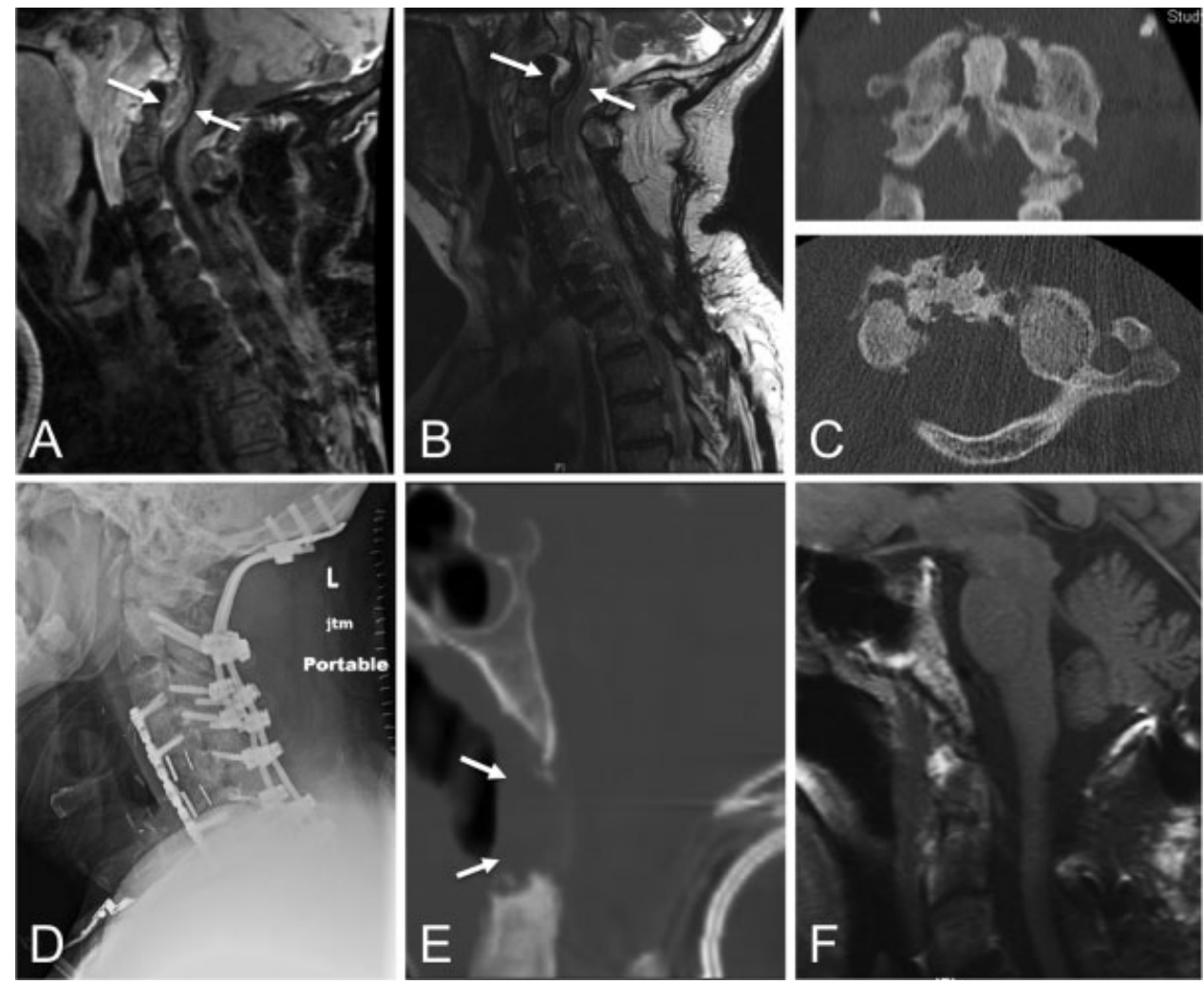

Fig. 2 Case 2: (A) Preoperative T1-weighted imaging with gadolinium demonstrates an enhancing collection centered at the atlantoaxial junction. (B) T2-weighted imaging shows heterogeneous signal intensity in the collection with posterior mass effect. (C) Coronal (upper panel), and axial (lower panel) CT cuts through the atlantoaxial joint suggestive of diseased bone. (D) Lateral X-ray demonstrating extension of the previous construct to the occiput. Also visible is the patient's previous anterior fusion. (E) Intraoperative CT confirming the extent of odontoid resection. (F) Postoperative T1-weighted image with gadolinium after resection demonstrating cervicomedullary decompression. CT, computed tomography.

\section{Intervention}

Preoperative CT imaging confirmed the presence of an adequate surgical corridor to the atlantoaxial junction via a transnasal approach. As such, a two-stage operation was planned to treat the presumed atlantoaxial osteomyelitis. Initial posterior stabilization was first extended to the occiput (-Fig. 2D). Two days later, after lumbar drain placement and setup of intraoperative navigation, a multidisciplinary anterior debridement was performed with computer-assisted stereotactic navigation as a joint operation involving otolaryngology and neurosurgery in a similar fashion to Case 1. Red rubber catheters were utilized for intermittent soft palate retraction. Curved and extended 3- and 4-mm coarse diamond drill bits were used to take down the arch of $\mathrm{C} 1$ and inferior aspect of the clivus exposing thick granulation tissue. Additional fibrotic inflammatory tissue and soft tissue edema were noted in the $\mathrm{C} 1-\mathrm{C} 2$ region, but no frank purulence was appreciated. Successful dural decompression was confirmed endoscopically and corroborated with intraoperative CT (-Fig. 2E). Although no CSF was seen, a dural defect was noted with exposed arachnoid. As such, an abdominal fat graft was placed against the dura along with Gelfoam and Tisseel. The indwelling lumbar drain was placed to drain at 15 $\mathrm{mL} / \mathrm{h}$ given the possibility of CSF leak from the basilar cistern. The patient was slow to wean off of the ventilator, but was extubated uneventfully on postoperative day 3. Postsurgical imaging confirmed optimal decompression of the cervicomedullary junction (-Fig. 2F). Pathology demonstrated granulation tissue and inflamed synovium, but interestingly all cultures remained negative throughout his hospital course, likely due to concurrent use of intravenous antibiotics during surgery. After consulting with the infectious disease team, antibiotics were discontinued and the patient was discharged home on postoperative day 7 .

\section{Follow-Up}

At 3-month follow-up, the patient reported complete resolution of his preoperative symptoms, denied any new symptoms of velopharyngeal insufficiency, Eustachian tube dysfunction, and his neurological examination was only notable for the new limited cervical range of motion consistent with occipitocervical fusion. On examination, the posterior cervical incision was well healed and his nasal endoscopy showed a widely remucosalized nasopharynx without pooling of secretions or edema.

\section{Discussion}

Vertebral osteomyelitis comprises only 2 to $4 \%$ of all cases of osteomyelitis and involvement of the craniocervical junction 
represents an especially rare subset of this acute inflammatory disease process. CSF fistula occurring in the setting of osteomyelitis is also quite atypical, but must be recognized in a timely manner-particularly in patients presenting with meningitis and/or immunosuppression. In this setting, Staphylococcus aureus is recognized as the most common causative microorganism ${ }^{2}$ although infection with Streptococcus ${ }^{9}$ and gram-negative organisms has been reported. As illustrated in this case series, notable risk factors for acute skull base osteomyelitis include diabetes mellitus, intravenous drug abuse, active immunosuppression, and hemodialysis. ${ }^{9}$

Although IV antibiotics can successfully treat this dire condition, in selected cases, acute surgical decompression may be required in cases of infected and degenerated vertebral bone causing cervicomedullary compression. Transoral decompression has been the "gold standard" procedure for anterior decompression of $\mathrm{C} 1$ and $\mathrm{C} 2$, however, there may be disadvantages to this approach, specifically in the setting of acute osteomyelitis, and immunocompromised with poor baseline health. ${ }^{1-9}$ These include a long surgical corridor with limited visualization, and limited manual/digital access beyond the oral cavity given dental, occlusal, and palatal anatomic constraints. Following a transoral approach, there is exposure of the surgical bed to saliva and oral contents with each swallow raising concern for impaired healing or infection, especially in the event of CSF leakage. Postoperative oropharyngeal swelling increases the concern for safe extubation, and often dictates consideration of tracheostomy and feeding tube placement. These concerns are again naturally exacerbated in immunocompromised patients with poor baseline health, as is frequently the case in those presenting with craniocervical osteomyelitis and ventral epidural abscess.

We and others have previously described the transnasal approach to the craniocervical junction for several etiologies. ${ }^{12-24}$ Advantages to the transnasal approach include outstanding endoscopic visualization of the full length of the deep surgical corridor created, favorable nasal mucosal wound healing, early advancement to an oral diet, and the reduced likelihood of prolonged postoperative intubation due to concerns of surgical site swelling. Disadvantages are primarily (1) anatomic, as the hard palate of the oral cavity limits endoscopic instrumentation below the $\mathrm{C} 2$ vertebral body, and (2) technical, given the need for multidisciplinary surgical expertise with endonasal neurosurgery and for specialized equipment required to access this distant skull base location. In addition to acute skull base osteomyelitis, we have previously also utilized the transnasal approach for decompression of patients with cervicomedullary tumor, rheumatoid arthritis, and degenerative pseudotumor. ${ }^{14,19}$ Postoperatively, both patients detailed herein were extubated and tolerated an oral diet 1 to 3 days after surgery, which may not be feasible until several days after a transoral approach.

Careful judgment must be employed in determining when transnasal versus transoral decompression is appropriate. Anatomic considerations were recently highlighted by the Rhoton group who compared the two approaches in 10 cadaveric specimens and proposed use of a combined approach when necessary. ${ }^{20}$ Following cervical fusion, our evaluation begins with a thin-cut CT scan and angiogram of the nasal cavity and craniocervical junction to fully delineate all critical neurovascular anatomy, and to ascertain whether the hard palate will markedly restrict inferior access to C2 (-Fig. 1D). In all cases, there will be bony deformation at the skull base, and important surgical landmarks, such as, the C1 tubercle may in fact be eroded, increasing the risk of inadvertent vertebral artery injury. We consistently utilize intraoperative stereotactic navigation with merged stereotactic MRI and CT angiogram sequences to attain reliable positional verification to mitigate this risk. ${ }^{14}$

Both of our patients were treated in a staged fashion with initial posterior stabilization. We are strong advocates that posterior stabilization should precede all considerations of anterior endonasal surgery. Arthrodesis at $\mathrm{C} 1 / 2$ was adequate for the first case. For the second patient, osteomyelitis involving the $\mathrm{C} 1$ lateral masses in conjunction with the patient's preexisting instrumentation necessitated extension of fixation to the occiput. Two days following the posterior stabilization, we utilized a transnasal approach for mandatory urgent debridement of diseased bone and infected tissue, achieving both decompression, surgical clearance of frankly diseased tissue, infection and inflammation, and pathologic diagnosis.

A large $1 \mathrm{~cm}$ dural tear occurred after successful decompression during the first case, and exposed arachnoid was visualized in the second case suggestive of elevated risk for postoperative CSF leakage despite no visualization of CSF. Although primary dural repair in the premedullary space is challenging, we successfully reconstructed even the $1-\mathrm{cm}$ defect using a multilayered onlay grafting technique consisting of DuraGen sheeting, abdominal fat, Gelfoam, fibrin glue, and biosorbable polyurethane sponge (Nasopore). Importantly, this reconstruction of the surgical defect did not produce secondary brainstem compression, and obviated the need for pedicled intranasal flap maneuvers.

Both patients had lumbar drains placed at the outset of each case, in anticipation of the need for CSF diversion following endonasal surgery in these acutely infected/inflamed surgical beds. Strategically, early lumbar drain placement at case start is readily performed, and obviates the need to reposition the patient in a lateral decubitus position after insetting a multilayered reconstruction. Our experience and that of others ${ }^{25}$ suggests that a drainage rate of at least $15 \mathrm{~mL} /$ $\mathrm{h}$ may be required in some cases to alleviate CSF leakage. Clinical judgment must be used to weigh the risks of postoperative CSF leakage against those incurred by CSF diversion. In these immunocompromised patients with likely suboptimal wound healing and dural defects in the premedullary space, we felt that drainage at $15 \mathrm{~mL} / \mathrm{h}$ was warranted. Although no adverse effects were observed in either patient, use of a lumbar drain requires vigilant monitoring for symptoms of intracranial hypotension, including depressed consciousness, especially when higher drainage rates are employed. ${ }^{26,27}$

In summary, this case series details the successful use of endoscopic transnasal anterior decompression to treat basilar invagination secondary to acute skull base osteomyelitis, and 
adds to the growing literature in support of the transnasal endoscopic approach as a safe and feasible means for decompressing the craniocervical junction, now also in the setting of active infection and inflammation in immunocompromised patients. Judicious patient selection, combined with sound clinical judgment, access to instrumentation and intraoperative imaging cannot be overemphasized. CSF leaks at this distant location anterior to the brainstem can also be successfully repaired with bolstered multilayered reconstructive materials without secondary neurologic consequences or impeding the goal of decompressing the ventral posterior fossa skull base.

\section{References}

1 Keogh S, Crockard A. Staphylococcal infection of the odontoid peg. Postgrad Med J 1992;68(795):51-54

2 Suchomel P, Buchvald P, Barsa P, Lukas R, Soukup T. Pyogenic osteomyelitis of the odontoid process: single stage decompression and fusion. Spine 2003;28(12):E239-E244

3 Ueda Y, Kawahara N, Murakami H, Matsui T, Tomita K. Pyogenic osteomyelitis of the atlas: a case report. Spine 2009;34(9): E342-E345

4 Wiedau-Pazos M, Curio G, Grüsser C. Epidural abscess of the cervical spine with osteomyelitis of the odontoid process. Spine 1999;24(2):133-136

5 Yang SY, Gao YZ. Clinical results of the transoral operation for lesions of the craniovertebral junction and its abnormalities. Surg Neurol 1999;51(1):16-20

6 Yau EL, Li K-K. Concomitant fungal and bacterial atlanto-axial osteomyelitis: a case report. J Orthop Surg (Hong Kong) 2010; 18(2):241-243

7 Young WF, Weaver M. Isolated pyogenic osteomyelitis of the odontoid process. Scand J Infect Dis 1999;31(5):512-515

8 Zigler JE, Bohlman HH, Robinson RA, Riley LH, Dodge LD. Pyogenic osteomyelitis of the occiput, the atlas, and the axis. A report of five cases. J Bone Joint Surg Am 1987;69(7):1069-1073

9 Reid PJ, Holman PJ. Iatrogenic pyogenic osteomyelitis of C-1 and C2 treated with transoral decompression and delayed posterior occipitocervical arthrodesis. Case report. J Neurosurg Spine 2007; 7(6):664-668

10 Crockard HA. Transoral surgery: some lessons learned. Br J Neurosurg 1995;9(3):283-293

11 Dickman CA, Locantro J, Fessler RG. The influence of transoral odontoid resection on stability of the craniovertebral junction. J Neurosurg 1992;77(4):525-530

12 Gempt J, Lehmberg J, Grams AE, Berends L, Meyer B, Stoffel M. Endoscopic transnasal resection of the odontoid: case series and clinical course. Eur Spine J 2011;20(4):661-666

13 Fraser JF, Anand VK, Schwartz TH. Endoscopic biopsy sampling of tophaceous gout of the odontoid process. Case report and review of the literature. J Neurosurg Spine 2007;7(1):61-64
14 Choudhri O, Mindea SA, Feroze A, Soudry E, Chang SD, Nayak JV. Experience with intraoperative navigation and imaging during endoscopic transnasal spinal approaches to the foramen magnum and odontoid. Neurosurg Focus 2014;36(3):E4

15 Hankinson TC, Grunstein E, Gardner P, Spinks TJ, Anderson RCE. Transnasal odontoid resection followed by posterior decompression and occipitocervical fusion in children with Chiari malformation Type I and ventral brainstem compression.J Neurosurg Pediatr 2010;5(6):549-553

16 Kassam AB, Snyderman C, Gardner P, Carrau R, Spiro R. The expanded endonasal approach: a fully endoscopic transnasal approach and resection of the odontoid process: technical case report. Neurosurgery 2005;57(1, Suppl):E213, discussion E213

17 Laufer I, Greenfield JP, Anand VK, Härtl R, Schwartz TH. Endonasal endoscopic resection of the odontoid process in a nonachondroplastic dwarf with juvenile rheumatoid arthritis: feasibility of the approach and utility of the intraoperative Iso- $C$ three-dimensional navigation. Case report. J Neurosurg Spine 2008;8(4):376-380

18 Leng LZ, Anand VK, Hartl R, Schwartz TH. Endonasal endoscopic resection of an os odontoideum to decompress the cervicomedullary junction: a minimal access surgical technique. Spine 2009; 34(4):E139-E143

19 Nayak JV, Gardner PA, Vescan AD, Carrau RL, Kassam ABSC, Snyderman $\mathrm{CH}$. Experience with the expanded endonasal approach for resection of the odontoid process in rheumatoid disease. Am J Rhinol 2007;21(5):601-606

20 Seker A, Inoue K, Osawa S, Akakin A, Kilic T, Rhoton AL Jr Comparison of endoscopic transnasal and transoral approaches to the craniovertebral junction. World Neurosurg 2010;74(6): 583-602

21 Iacoangeli M, Gladi M, Alvaro L, Di Rienzo A, Specchia N, Scerrati M. Endoscopic endonasal odontoidectomy with anterior C1 arch preservation in elderly patients affected by rheumatoid arthritis. Spine J 2013;13(5):542-548

22 Patel AJ, Boatey J, Muns J, et al. Endoscopic endonasal odontoidectomy in a child with chronic type 3 atlantoaxial rotatory fixation: case report and literature review. Childs Nerv Syst 2012;28(11): 1971-1975

23 Yu Y, Wang X, Zhang X, et al. Endoscopic transnasal odontoidectomy to treat basilar invagination with congenital osseous malformations. Eur Spine J 2013;22(5):1127-1136

24 Yu Y, Hu F, Zhang X, Ge J, Sun C. Endoscopic transnasal odontoidectomy combined with posterior reduction to treat basilar invagination: technical note. J Neurosurg Spine 2013;19(5): 637-643

25 Açikbaş SC, Akyüz M, Kazan S, Tuncer R. Complications of closed continuous lumbar drainage of cerebrospinal fluid. Acta Neurochir (Wien) 2002;144(5):475-480

26 Loya JJ, Mindea SA, Yu H, Venkatasubramanian C, Chang SD, Burns TC. Intracranial hypotension producing reversible coma: a systematic review, including three new cases. J Neurosurg 2012;117(3): 615-628

27 Wang K, Liu Z, Chen X, Lou M, Yin J. Clinical characteristics and outcomes of patients with cerebral herniation during continuous lumbar drainage. Turk Neurosurg 2013;23(5):653-657 